\title{
Do Lions Have Manes? For Children, Generics Are About Kinds Rather Than Quantities
}

\author{
Amanda C. Brandone \\ Lehigh University \\ Sarah-Jane Leslie \\ Princeton University
}

\author{
Andrei Cimpian \\ University of Illinois at Urbana-Champaign \\ Susan A. Gelman \\ University of Michigan
}

\begin{abstract}
Generic statements (e.g., "Lions have manes") make claims about kinds (e.g., lions as a category) and, for adults, are distinct from quantificational statements (e.g., "Most lions have manes"), which make claims about how many individuals have a given property. This article examined whether young children also understand that generics do not depend purely on quantitative information. Five-year-olds $(n=36)$ evaluated pairs of questions expressing properties that were matched in prevalence but varied in whether adults accept them as generically true (e.g., "Do lions have manes?" [true] vs. "Are lions boys?" [false]). Results demonstrated that children evaluate generics based on more than just quantitative information. Data suggest that even young children recognize that generics make claims about kinds.
\end{abstract}

A central task of early childhood is to learn broad generalizations about categories in the world. Children must learn that lions have manes, milk builds strong bones, and stoves are hot. Generic sentences such as "Lions have manes" provide an important means of conveying such knowledge (Gelman, 2003). Generics make claims about kinds (e.g., lions as a category) rather than individuals (e.g., the lions at the zoo) and express generalizations about shared properties of category members (Carlson, 1977; Carlson \& Pelletier, 1995). Generics also link to core properties, as they express predicates that are relatively enduring (not transient), timeless (not contextually bound), and inherent (not accidental) (Carlson \& Pelletier, 1995; Prasada, 2000; see also Prasada \& Dillingham, 2006). Generics appear frequently in natural speech, including conversation with young children (e.g., Gelman, Goetz, Sarnecka,

This research was supported by an NSF Graduate Research Fellowship to Amanda C. Brandone, University of Illinois funds to Andrei Cimpian, Princeton University funds to Sarah-Jane Leslie, and NICHD Grant HD-36043 and NSF Grant BCS0817128 to Susan A. Gelman. We are grateful to the children, parents, and teachers at the UM Children's Center for participating in this research. We also thank Kristin Lang, Jenna Hedglen, Ben Boldt, Melissa Yako, and the Concepts and Theories in Human Development and UIUC Cognitive Development Labs for assistance in data collection.

Correspondence concerning this article should be addressed to Amanda Brandone, Department of Psychology, Lehigh University, Bethlehem, PA 18015. Electronic mail may be sent to acb210@lehigh.edu.
\& Flukes, 2008), and they have been hypothesized to play a central role in human reasoning (Gelman, 2003; Prasada, 2000). Nevertheless, an issue that remains unresolved concerns how the meaning of these utterances is represented.

Within the linguistic and philosophical literatures, there has been considerable debate over how to characterize the semantics of generics (for a review, see Leslie, 2008). Leslie $(2007,2008)$ has argued that the truth of a generic statement does not depend on how many kind members possess the property in question; there is no straightforward mapping between statistical facts about the world and judgments about generics (see also Carlson, 1977; Carlson \& Pelletier, 1995; cf. Prasada \& Dillingham, 2006). Consider these examples. "Lions have manes" is true, but "Lions are male" is false, even though only male lions ever possess manes. "Mosquitoes carry the West Nile Virus" is true, even though less than $1 \%$ of mosquitoes are actually infected. Conversely, "People are right-handed" is false, despite the fact that the vast majority of individuals are right-handed. Examples such as these demonstrate that when deciding on the truth value

(C) 2012 The Authors

Child Development (C) 2012 Society for Research in Child Development, Inc. All rights reserved. 0009-3920/2012/8302-0004

DOI: $10.1111 / j .1467-8624.2011 .01708 . x$ 
of a generic, people rely on more than just statistical information.

Recent empirical work confirms that, for adults, generic meaning does not reduce to quantificationthat is, to considerations of how many members of the kind have the property. Two main findings have emerged from this research. First, adults' acceptance of generics is not accounted for by the prevalence of the predicated properties alone. (It is only in certain circumscribed cases that statistical frequency is sufficient to make a generic true; Prasada \& Dillingham, 2006, 2009.) For example, Khemlani and colleagues (Khemlani, Leslie, \& Glucksberg, 2009; Khemlani, Leslie, Glucksberg, \& Rubio-Fernandez, 2007) showed that adults judged some generics such as "Ducks lay eggs" and "Mosquitoes carry the West Nile virus" to be true despite knowing that a large percentage of the kinds lacks the predicated properties; however, they rejected other generics such as "Books are paperbacks" and "Canadians are right-handed" despite knowing that most members of those kinds have the predicated properties. Furthermore, Cimpian, Brandone, and Gelman (2010) found that adults were willing to accept even some novel generics (e.g., "Lorches have purple feathers") at low prevalence levels (below 50\%).

Second, research has shown that adults' acceptance of generics reflects biases in their conceptual representations. For example, Cimpian, Brandone, et al. (2010) found that generic predications of properties that were described as distinctive or dangerous (e.g., venomous scales) were accepted more often than generic predications of other similar properties (e.g., shiny scales), presumably because properties that are either distinctive or dangerous to humans are privileged in our kind representations (see also Leslie, 2007, 2008). Similarly, Cimpian, Gelman, and Brandone (2010) and Gelman and Bloom (2007) showed that considerations about the nature and origin of the predicated properties (e.g., whether they are innate vs. acquired) are sufficient, independently of prevalence, to determine how adults reason about generic sentences.

Together, these data suggest that, at least for adults, generics do not reduce to quantificational statements. An important remaining question is whether this is also the case for children: Do children understand generic meaning as distinct from quantification? This question has important implications for broader theoretical questions regarding the nature of children's concepts. Some have argued that as young children build their lexical and conceptual repertoires, they are guided by abstract conceptual knowledge (e.g., ontology, animacy, function), including a conceptual distinction between individuals and kinds (e.g., Carey, 1985, 2009; Gelman, 2003; Gelman \& Brandone, 2010; Gopnik \& Meltzoff, 1997; Wellman \& Gelman, 1998). However, others have proposed a more bottom-up process of lexical and conceptual development in which perceptual features of the environment get linked to concrete linguistic features of the input via associative learning (e.g., Colunga \& Smith, 2005; Sloutsky \& Fisher, 2004; Sloutsky, Kloos, \& Fisher, 2007; Smith, Jones, \& Landau, 1996). These theoretical perspectives offer distinct proposals for how children might interpret generics. If children's concepts go beyond the available evidence and incorporate abstract knowledge, then children may be able to interpret generics as being about abstract kinds and as distinct from statements about how many members of the kind have the relevant property. If, on the other hand, children's cognition is limited to statistical computations and associative learning, then children may instead interpret generics as synonymous with quantified statements.

Much of the recent evidence regarding children's production and comprehension of generics is suggestive of the possibility that generics are not equivalent to statements about frequency even for young children. Children as young as $2 \frac{1}{2} 2$ years of age produce generics (Gelman, Goetz, et al., 2008; Gelman, Waxman, \& Kleinberg, 2008) and do so more often when the context encourages a focus on kinds (Brandone \& Gelman, 2009; Gelman, Goetz, et al., 2008; Gelman, Waxman, et al., 2008). For example, preschoolers produce more generics when talking about animals than artifacts (Brandone \& Gelman, 2009; Gelman, Goetz, et al., 2008; Gelman, Waxman, et al., 2008)—consistent with evidence suggesting that animal categories are more richly structured and "kind-like" than artifact categories (e.g., Keil, 1989). These data suggest that children understand generics to be making claims about kinds. However, production data are ambiguous: When children produce a generic (e.g., "Birds fly"), we cannot be certain whether they are referring to the kind or to a quantified set (e.g., all or most birds).

So far, the evidence from comprehension studies also cannot definitively rule out the possibility that children understand generics as quantificational. Preschoolers seem to recognize that generics (a) refer beyond individuals in the immediate context (Cimpian \& Markman, 2008; Cimpian, Meltzer, \& Markman, 2011; Gelman \& Bloom, 2007; Gelman \& Raman, 2003), (b) are broad in scope but allow for 
exceptions (Chambers, Graham, \& Turner, 2008; Gelman \& Bloom, 2007), and, more specifically, (c) imply scope that is intermediate between "all" and "some" (Hollander, Gelman, \& Star, 2002). Although these studies demonstrate sophisticated understanding of generics in preschoolers, their results are still compatible with the possibility that children interpret generics as equivalent to quantificational statements about individuals (e.g., perhaps as equivalent to "most" statements).

Thus, existing data provide suggestive, but not conclusive, evidence that children do not interpret generics as making purely quantificational claims. In the current experiments, we examine this question directly. Specifically, we ask: Do young children interpret generics as reflecting only statistical facts about the world? Or, as in the case of adults, do judgments about the truth of generics depend on additional information?

\section{Study 1}

We compared children's judgments about pairs of statements that, although matched in prevalence, vary in their acceptability as generics. Consider, for example, "Lions have manes" versus "Lions are male." Since only male lions have manes, "Lions have manes" applies to at most as many lions as "Lions are male." Yet, despite the similar underlying statistics of these two statements, only "Lions have manes" seems acceptable, arguably because having manes is a characteristic property of the kind lion, whereas being male is not. The question of what makes a property characteristic of a kind is an exceedingly complex one (see Leslie, 2007, 2008; Prasada \& Dillingham, 2006, 2009). For current purposes, a property was deemed "characteristic" if it involved (a) a salient physical property (e.g., "Lions have manes"), (b) a means of gestation (e.g., "Birds lay eggs"), or (c) a means of nurturing the young (e.g., "Pigs give milk to their babies"). A property was deemed "noncharacteristic" if it involved attributing a gender to the kind (e.g., "Lions are male"). Theoretically, this does not exhaust all the possibilities for characteristic versus noncharacteristic properties; nevertheless, these were the guidelines selected to operationalize characteristic and noncharacteristic properties in the current study.

The previous example illustrates the research strategy used in this study: We compared the likelihood that participants would accept as true each of two kinds of statements presented in generic form: (a) those for which predicates are characteristic of the kind yet true of only one gender (e.g., "Lions have manes") and (b) those for which predicates are not characteristic of the kind yet also true of only one gender (e.g., "Lions are boys"). (Note that because of the age of our participants, we substituted the terms boys and girls for males and females. Pilot testing confirmed that children understood these terms as referring to the gender of the animals and did not interpret them to mean human boys and human girls.)

Pairs of characteristic and noncharacteristic statements (e.g., "Lions have manes" vs. "Lions are boys") were chosen such that, within each pair, the property predicated in the noncharacteristic statement (e.g., being male) served as a precondition for the property predicated in the characteristic statement (e.g., having a mane) and thus applied to a superset of the animals to which the characteristic property applied. To ensure that participants were aware of these inclusion relations, we tested their knowledge of the gender requirements for the properties predicated in the characteristic statements (e.g., "Do boy lions have manes?" "Do girl lions have manes?"). Only items on which participants demonstrated requisite knowledge (e.g., that only boy lions have manes) were analyzed.

By equating the prevalence of the information expressed in the two types of statements and varying whether or not the predicated property was characteristic of the kind, we could distinguish responses driven by prevalence information from responses driven by knowledge about kinds. If children interpret generics as reflecting purely statistical facts about the world, they should be equally likely to accept the characteristic and noncharacteristic statements because the properties predicated in each are roughly equivalent in prevalence. If, on the other hand, children interpret generics as claims about kinds like adults do, then they should be more likely to accept the characteristic than the noncharacteristic statements. We predicted that children's understanding of generics would not be driven solely by statistical facts about the world; thus, acceptance of the characteristic items should be greater than acceptance of the noncharacteristic items.

\section{Method}

\section{Participants}

Fourteen children ( 7 males, 7 females; $M=$ 5.36 years, $S D=.52$ ) and 14 undergraduates (5 males, 9 females) participated. Two additional children were excluded because they did not know any 
of the gender properties. An additional 28 undergraduates participated in pretesting (see below). Children were recruited from schools in a Midwestern city. Undergraduates were recruited from the subject pools at two large public universities. Participants were predominantly European American and from middle-income homes.

\section{Materials and Procedure}

Part 1. Participants evaluated the truth or falsity of 20 items presented in question form (e.g., Do lions have manes?) in one of four semirandom orders. Participants responded by answering definitely yes, definitely no, or a little yes, a little no. The a little yes, a little no response was included to increase the sensitivity of the measure by creating a midpoint response, which allowed participants to show doubt about an item without completely rejecting it. Answer choices corresponded to pictures of a thumb pointing up, down, and sideways. To motivate the task, children were asked to help an alien learn about animals on Earth. Children responded by pointing to the thumb pictures. Undergraduates responded to written questions by circling the appropriate thumb images.

Items consisted of six characteristic and noncharacteristic item pairs (see Table 1). Within each pair, the characteristic item expressed a property that is characteristic of the target kind yet present in only one gender (e.g., "Do lions have manes?"). This gender information then served as the predicate for the corresponding noncharacteristic item (e.g., "Are lions boys?"). Because the property predicated in each noncharacteristic item was a precondition for the property predicated in the corresponding characteristic item, characteristic and noncharacteristic properties were roughly equivalent in prevalence (see the Adult Pretests section below). Eight filler items with clear, obvious answers were also included (four characteristic, four noncharacteristic; see Table 1) to screen out children who did not understand the task. The filler items expressed properties that are also considered characteristic (e.g., "Do tigers have stripes?") or noncharacteristic (e.g., "Do dogs have wings?") of their target kind; however, because in all cases the properties in these filler items are present or absent in both genders and thus do not allow for prevalence-matched comparison items, these items were not included in our central analyses. Children responded correctly to the vast majority of the filler items $(M=97.3 \%)$. Every child answered all or all but one of the filler items correctly.
Table 1

Study 1: Part 1 Items

\begin{tabular}{|c|c|}
\hline Item type & Item \\
\hline Characteristic & $\begin{array}{l}\text { Do birds lay eggs? } \\
\text { Do pigs give milk to their babies? } \\
\text { Do horses grow their babies in their } \\
\text { tummies? } \\
\text { Do lions have manes? }^{\mathrm{a}} \\
\text { Do deer have antlers? }^{\mathrm{a}} \\
\text { Do goats have horns? }^{\mathrm{a}}\end{array}$ \\
\hline Noncharacteristic & $\begin{array}{l}\text { Are birds girls? }^{\mathrm{a}} \\
\text { Are pigs girls? }^{\mathrm{a}} \\
\text { Are horses girls? } \\
\text { Are lions boys? }^{\mathrm{a}} \\
\text { Are deer boys? }^{\text {Are goats boys? }}\end{array}$ \\
\hline Filler & $\begin{array}{l}\text { Do tigers have stripes? } \\
\text { Do cats have tails? }^{\mathrm{a}} \\
\text { Do kangaroos hop? } \\
\text { Do cows say moo? } \\
\text { Do dogs have wings? }^{\mathrm{a}} \\
\text { Do snakes have feet? } \\
\text { Do hamsters fly } \\
\text { Do fish say woof? }\end{array}$ \\
\hline
\end{tabular}

${ }^{\mathrm{a}}$ Indicates items used in Study 2.

Part 2. In this part, we tested participants' knowledge of the gender requirements for the characteristic properties (e.g., that only male lions have manes). Participants were asked about 10 properties: 6 characteristic properties (from Part 1) that apply to individuals of only one gender, 2 filler properties that apply to individuals of both genders (e.g., both boy and girl tigers have stripes), and 2 filler properties that do not apply to individuals of either gender (e.g., neither boy nor girl dogs have wings). Participants were asked separate, sequential questions about whether these properties apply to the males and females of the species (e.g., "Do boy lions have manes? Do girl lions have manes?"). Question pairs were presented in one of four semirandom orders. Whether the female or male version of the question appeared first was counterbalanced across subjects.

Participants responded by answering definitely yes, definitely no, or a little yes, a little no. Children pointed to indicate their answer to a second alien's questions about "boy animals and girl animals." Undergraduates circled the appropriate thumb image. If participants responded incorrectly to a Part 2 question, that item pair was excluded from their Part 1 data. Responses were considered incorrect if participants answered either of the 
questions incorrectly (e.g., saying that boy lions do not have manes or that girl lions do). To be conservative, the intermediate answer choice was counted as a negative response. Both children and undergraduates knew the majority of the facts tested in Part $2\left(M_{\text {Children }}=4.07\right.$ of 6 possible; $\left.M_{\text {Undergraduates }}=4.93\right)$.

Adult pretests. To confirm that the predicates of the characteristic and noncharacteristic items are considered equally prevalent, we conducted two pretests with separate groups of undergraduates. In the first, 16 undergraduates estimated the prevalence of the properties (e.g., having manes, being male) used in the main task. For example, participants were told, "It is estimated that there are roughly 32,000 individual lions in the world. Please estimate what percentage of those individual lions have manes." Participants estimated the characteristic and noncharacteristic properties to be roughly equal in prevalence, $F(6,10)=0.39, p=.87$, applying to an average of $54.3 \%$ and $49.6 \%$ of category members, respectively.

In the second pretest, a separate group of 16 undergraduates performed a comparative judgment for each item pair. They were asked, for example, "Which do you think there are more of? (a) Boy lions; (b) Lions that have manes; (c) They are equal." Across all item pairs, the dominant response was "They are equal." Participants selected this response on $67.7 \%$ of trials, significantly more often than expected by chance $(33.3 \%), t(15)=3.54, p=.003$. Participants selected the characteristic response (e.g., "Lions that have manes") on 6.3\% of trials (significantly less likely than expected by chance), $t(15)=12.84, p<.001$, and the noncharacteristic response (e.g., "Boy lions") on $26.0 \%$ of trials (at chance levels), $t(15)=0.72, p=.49$. Overall, pretest results confirmed that adults consider the characteristic and noncharacteristic properties to be roughly equal in prevalence.

\section{Results}

Our central analysis involved the proportion of definitely yes responses for the characteristic and noncharacteristic items. We conducted a 2 (item type: characteristic, noncharacteristic; within subject) $\times 2$ (age group: children, undergraduates; between subjects) analysis of variance (ANOVA). The dependent variable was a proportion score based on the number of times participants selected the definitely yes response out of the total number of item pairs on which they demonstrated requisite knowledge in Part 2.

Results revealed the predicted main effect of item type, $F(1,26)=34.22, p<.001, \eta_{\mathrm{p}}{ }^{2}=.57$. As shown in Table 2, both children, $F(1,13)=11.27$, $p=.002, \eta_{\mathrm{p}}{ }^{2}=.30$, and undergraduates, $F(1,13)$ $=24.16, p<.001, \eta_{\mathrm{p}}{ }^{2}=.48$, gave significantly more definitely yes responses for characteristic than noncharacteristic items. (The proportion of definitely no and a little yes, a little no responses are also displayed in Table 2.) This pattern of results held for 10 of 14 children, $p=.012$, and 11 of 14 undergraduates, $p=.006$ (sign tests). Results also revealed a nonsignificant trend toward an effect of age group, $F(1,26)=3.23, p=.084, \eta_{\mathrm{p}}^{2}=.11$, with children tending to choose definitely yes more often than undergraduates $(M s=0.61$ and 0.43 , respectively).

Additional analyses were conducted to compare the proportion of definitely yes responses against rates expected by chance (.33, Table 2$)$. As predicted, for the characteristic items both children, $t(13)=6.20, p<.001$, and undergraduates, $t(13)=$ $4.37, p=.001$, selected the definitely yes response at rates greater than expected by chance. This was not the case for the noncharacteristic items. Children, $t(13)=1.41, p=.18$, and undergraduates, $t(13)=$ $1.24, p=.24$, selected the definitely yes response for these items at chance levels.

Table 2

Mean Proportion (Standard Deviation) of Responses by Response Type, Age Group, and Item Type

\begin{tabular}{|c|c|c|c|c|c|}
\hline \multirow[b]{2}{*}{ Study } & \multirow[b]{2}{*}{ Age group } & \multirow[b]{2}{*}{ Item type } & \multicolumn{3}{|c|}{ Proportion of responses $(S D)$} \\
\hline & & & Definitely yes & A little yes, A little no & Definitely no \\
\hline \multirow[t]{4}{*}{1} & \multirow[t]{2}{*}{ Adult } & Characteristic & $.65(.28)^{*}$ & $.35(.28)$ & $.00(.00)^{*}$ \\
\hline & & Noncharacteristic & $.21(.36)$ & $.78(.35)^{*}$ & $.01(.04)^{*}$ \\
\hline & \multirow[t]{2}{*}{ Children } & Characteristic & $.76(.26)^{*}$ & $.17(.24)^{*}$ & $.07(.19)^{*}$ \\
\hline & & Noncharacteristic & $.46(.35)$ & $.47(.37)$ & $.07(.20)^{*}$ \\
\hline \multirow[t]{2}{*}{2} & \multirow[t]{2}{*}{ Children } & Characteristic & $.86(.33)^{*}$ & $.03(.10)^{*}$ & $.11(.30)^{*}$ \\
\hline & & Noncharacteristic & $.29(.45)$ & $.71(.45)^{*}$ & $.00(.00)^{*}$ \\
\hline
\end{tabular}

*Indicates $p<.05$ for comparisons against chance (.33). 


\section{Discussion}

Study 1 addressed the question of whether young children equate generics with quantification by comparing children's true and false judgments about pairs of statements that, although matched in prevalence, vary in whether or not they are considered acceptable generics by adults. Results showed that children were more likely to accept generics that express characteristic than noncharacteristic properties. These findings suggest that young children do not interpret generics as statements about the number or proportion of individual category members that possess a particular property. Instead, even young children recognize that generics make claims about kinds.

The argument that children do not interpret generics as equivalent to quantification rests on the finding that children were less likely to reply definitely yes to the noncharacteristic items. We argue that children did so because they (a) recognize that generics make claims about kinds and (b) do not consider the noncharacteristic items to be true of their kinds. However, there may be alternative reasons why children were less likely to accept the generics that involved noncharacteristic properties. One possibility is that children's responses were influenced by the relative novelty of the noncharacteristic stimuli. Children may be less likely to have heard statements expressing the noncharacteristic properties of the target categories than statements expressing their characteristic properties, which may in turn make children less likely to agree with these less familiar items. Although a stringent test of this possibility would require a study with novel categories, at least one aspect of the current study suggests that novelty effects cannot account for children's responses. Consider the filler items in Part 2 expressing properties that are characteristic of both genders (e.g., "Do boy/girl tigers have stripes?"). It is unlikely that children have previously heard statements expressing these properties (because the properties do not vary by gender and; thus, parents would be unlikely to talk about the genders separately). Nevertheless, children responded definitely yes on these items $78.6 \%$ of the time. The fact that children consistently gave definitely yes responses on questions they have likely not heard before casts doubt on the possibility that the novelty of the noncharacteristic stimuli led to their low acceptance levels.

Another possibility is that children provided fewer definitely yes and more a little yes, a little no responses on the noncharacteristic items because they were confused by the structure of these questions ("Are Xs Ys?"). To explore this possibility, we tested an additional 10 children $(M=5.19$ years $)$ on a similar task in which participants responded to questions that were structurally equivalent to the noncharacteristic items but varied in whether their correct answer was yes or no. This task included six items each for which the correct answer was (a) definitely yes (e.g., "Are princesses girls?" "Are daddy bears boys?"), (b) definitely no (e.g., "Are ladies boys?" "Are mommy ducks boys?"), and (c) a little yes, a little no (e.g., "Are kids girls?" "Are baby birds boys?"), for a total of 18 items. We reasoned that if children's responses to the noncharacteristic items were driven by confusion with the structure of these questions, then children should encounter the same difficulty on this task.

This was not the case. Although all the items in the control task were structurally analogous to the noncharacteristic items in the main study, children responded appropriately on an average of $78.0 \%$ of trials. Importantly, children had no trouble responding definitely yes where appropriate, doing so on $82.4 \%$ of items for which it was the correct response. These data suggest that children's low level of definitely yes responses to the noncharacteristic items on the central task was not due to the unusual structure of these items. Note that this control task also speaks against two additional concerns. First, the animal items (e.g., "Are daddy bears boys?") rule out the possibility that children interpreted questions such as "Are lions boys?" to mean "Are lions human boys?" (This possibility was remote anyway, as children would presumably have favored definitely no rather than a little yes, a little no if they had interpreted the questions in this way.) Second, this task rules out the possibility that children had more trouble responding to the noncharacteristic items because they had difficulty dealing with relations between sets (e.g., relations between the set of lions, boys, and boy lions; see Markman, 1989; Piaget, 1952).

A final alternative explanation we considered was that participants may have responded differently to the characteristic and noncharacteristic items as a result of semantic differences due to how the items were framed. All of the characteristic items either used the verb to have to attribute a physical feature to the kind (e.g., "Do lions have manes?"), or else used a verbal predicate to attribute a habitual action to the kind (e.g., "Do birds lay eggs?"). In contrast, all of the noncharacteristic items used nominal predicates (i.e., the verb to $b e+$ count noun) to attribute a gender to the kind 
(e.g., "Are lions boys?" "Are birds girls?"). This difference in syntactic frames is potentially problematic if statements about what a kind is carry different implications than statements about what a kind has or does.

There is some evidence to suggest that this might be the case. In particular, several studies have shown that using nominal versus verbal predicates to describe a characteristic of an individual influences judgments about the permanence of that characteristic. For example, Gelman and Heyman (1999) showed that 5-year-olds judge novel personal characteristics as more stable over time and context when they are expressed using a nominal predicate (e.g., "She is a carrot-eater") than when they are expressed by a verbal phrase (e.g., "She eats carrots whenever she can"; see also Cimpian, Arce, Markman, \& Dweck, 2007; Markman, 1989). Likewise, Reynaert and Gelman (2007) showed that adults judge physical and mental illnesses described using nominal predicates (e.g., "He is a baxtermic" and "He is baxtermic") as more permanent and stable than those described using verbal predicates (e.g., "He has baxtermia"). Thus, when describing characteristics of an individual, nominal and verbal predicates seem to carry different implications.

Of particular interest here is the question of whether statements about what a kind is versus what a kind has or does require different amounts of statistical evidence to be accepted as true. Specifically, given the permanence and centrality that is implied when properties are described using nominal predicates, it may be that more statistical evidence is required to agree with a statement about what a kind is than to agree with a statement about what a kind has or does (see Gilson \& Abelson, 1965). On this view, participants may have been less likely to endorse the noncharacteristic than the characteristic items not because of differences in whether or not the predicated properties are viewed as characteristic of their kinds, but rather because greater statistical evidence is necessary to endorse statements about what a kind is. To address this possibility, in Study 2 we examined children's responses to characteristic and noncharacteristic item pairs that are both presented with nominal predicates using the verb to $b e$.

\section{Study 2}

As in Study 1, we examined children's endorsements of items expressing properties that are characteristic of the kind yet true of only one gender, as well as of items that are not characteristic of the kind yet also true of only one gender. However, to control for the possibility that the item effects observed in Study 1 were driven by semantic differences due to how the characteristic and noncharacteristic items were framed (about what a kind is vs. what a kind has or does), in this study both the characteristic and noncharacteristic items were framed in terms of what the kind is. For example, to match the noncharacteristic item "Are lions boys?" the characteristic item "Do lions have manes?" was replaced with "Are lions animals with manes?" If, as argued, children's responses in Study 1 were driven by whether or not the predicated properties are viewed as characteristic of their kinds, then children should again be more likely to endorse generics that express characteristic than noncharacteristic properties, even when both types of items are presented with nominal predicates using the verb to $b e$.

\section{Method}

\section{Participants}

Twelve children (5 males, 7 females; $M=$ 5.10 years, $S D=.47)$ participated. Four additional children were excluded because they did not know any of the gender properties. The children, all of whom were recruited in a small Midwestern city, were predominantly European American and came from a range of socioeconomic backgrounds.

\section{Materials and Procedure}

Part 1. Children evaluated the truth or falsity of 12 items. Eight of the items were based on the four characteristic and noncharacteristic item pairs with which children from Study 1 were most familiar (see Table 1 for a full list). In addition, there were 4 filler items (2 characteristic, 2 noncharacteristic). Every child answered all or all but one of the filler items correctly. Importantly, all items used nominal predicates using the verb to be (e.g., characteristic: "Are lions animals with manes?"; noncharacteristic: "Are lions boys?"; filler: "Are tigers animals with stripes?"). All other aspects of the design were identical to Study 1.

Part 2. Part 2 was designed to test participants' knowledge of the gender requirements for the characteristic properties in Part 1 (e.g., that only male lions have manes). This section was identical in structure to Part 2 of Study 1; however, only the four characteristic properties from Part 1 and four filler items were examined. If participants 
responded incorrectly to a Part 2 question, that item pair was excluded from their Part 1 data. Children demonstrated knowledge of the gender requirements for roughly half of the properties tested ( $M=1.83$ of 4 possible). (It is unclear why children did not respond appropriately to more of the Part 2 items. The most common error was choosing definitely yes in response to questions about both male and female category members $[M=1.38$ of 4 possible], potentially suggesting that these participants, who were somewhat younger than those in Study 1 , had not yet learned how males and females differ on some of the characteristic properties.)

\section{Results}

Our central analysis involved the proportion of definitely yes responses for the characteristic and noncharacteristic items. We conducted an ANOVA with item type (characteristic, noncharacteristic) as a within-subject variable. The dependent variable was a proportion score based on the number of times participants selected the definitely yes response out of the total number of item pairs on which they demonstrated requisite knowledge in Part 2. Results revealed the predicted main effect of item type, $F(1,11)=17.33, p=.002, \eta_{\mathrm{p}}{ }^{2}=.61$. As shown in Table 2 , children gave significantly more definitely yes responses for characteristic than noncharacteristic items. This pattern held for 8 of 12 children, $p=.008$ (sign test).

Additional analyses were conducted to compare the proportion of definitely yes responses against rates expected by chance (.33; see Table 2). As predicted, for the characteristic items children selected the definitely yes response at rates greater than expected by chance, $t(11)=5.54, p<.001$. In contrast, children selected the definitely yes response for the noncharacteristic items at chance levels, $t(11)=-0.30, p=.77$.

\section{Discussion}

The purpose of this study was to evaluate the possibility that participants were more likely to agree with the characteristic than the noncharacteristic items in Study 1 because of semantic differences due to how these items were framed (i.e., as claims about what a kind has or does versus claims about what a kind is). Thus, in Study 2, children were asked to judge characteristic and noncharacteristic items that were both framed in terms of what the kind is (e.g., "Are lions animals with manes?" vs. "Are lions boys?"). We found that even when the characteristic and noncharacteristic items were framed in the same way, children were still significantly more likely to endorse generics that express characteristic than noncharacteristic properties. Together with the results of Study 1, these findings lend further support to our claim that when deciding on the truth value of a generic statement, what matters most is not the statistical prevalence of the property, but rather whether or not that property is viewed as characteristic of the kind.

\section{General Discussion}

This article explored whether young children understand generic meaning as distinct from quantification. We compared children's responses to questions about properties that, although roughly equivalent in prevalence (each applying to approximately $50 \%$ of kind members), differed in whether or not they are considered by adults to be generically true of the kind (e.g. "Do lions have manes?" or "Are lions animals with manes?" vs. "Are lions boys?"). As predicted, children were more likely to accept generics that express characteristic than noncharacteristic properties. These data expand upon the growing literature concerning children's comprehension of generics (e.g., Cimpian \& Markman, 2008; Gelman \& Bloom, 2007; Gelman \& Raman, 2003; Hollander et al., 2002) in two ways. First, our findings suggest that children recognize that generic facts can be considered true even if they are not true of all or even most members of a category (e.g., "Lions have manes"). Second, our data suggest that children recognize that some generic statements can be considered untrue even if they express properties that are fairly frequent (e.g., "Lions are boys"). Overall, the data presented here provide the first direct evidence that, like adults, young children do not interpret generics as statements about the number or proportion of individual category members that possess a particular property. Our data reveal instead that, even for young children, generics make claims about kinds.

These findings also speak to an ongoing debate about the nature of children's concepts. There is now substantial evidence to suggest that adults' concepts cannot be characterized by statistical information alone; they are better characterized as theory-like explanatory structures that incorporate not only knowledge about shared features (including nonobvious ones) but also causal-explanatory links between those features (e.g., Ahn, Flanagan, 
Marsh, \& Sanislow, 2006; Murphy, 2002; Rips \& Collins, 1993). The data presented here are consistent with the notion that the concepts of young children also consist of more than just statistical regularities (see also Gelman, 2003; Gopnik \& Schulz, 2004; Keil, 2006). Young children's interpretation of generic sentences reveals that they treat certain categories, including the animal categories examined here, as abstract generic kinds.

Of course, both statistical information and abstract conceptual knowledge likely play a role in children's reasoning about kinds (e.g., Prasada \& Dillingham, 2006, 2009). In fact, the current experiments provide some evidence that both children and adults also recognize and consider the role of prevalence information in evaluating the truth of generic statements. Consider participants' responses to the noncharacteristic items (e.g., "Are lions boys?"). Both children and adults avoided the definitely no response on these items in favor of the less definitive a little yes, a little no answer choice. This response may reflect participants' awareness that the noncharacteristic properties are true of at least some individuals within the target kinds. By selecting the a little yes, a little no response, participants were able to convey their knowledge of the prevalence of the noncharacteristic properties while still denying that these properties characterize the kind. This pattern of results is consistent with the view that although children do not rely on prevalence considerations alone when evaluating statements about kinds, they nonetheless do not ignore the statistical evidence. An important question for future research is how children's statistical learning mechanisms (e.g., Saffran, Aslin, \& Newport, 1996) interact with their abstract conceptual knowledge and their finely tuned causal reasoning abilities (e.g., Gopnik \& Schulz, 2007) to support conceptual development (see Sobel \& Kirkham, 2007; Tenenbaum, Griffiths, \& Kemp, 2006).

An additional important question this work raises concerns the nature of the differences between properties that can and cannot be truthfully predicated of a kind. The question of what makes a property generically true of a kind does not yet have a precise answer. According to one view (Prasada \& Dillingham, 2006, 2009), there are two circumstances under which it may be legitimate to predicate a property of an entire kind: (a) when a property is highly prevalent among kind members and (b) when a property has a "principled connection" with the kind-that is, when instances possess the feature by virtue of being the kinds of things they are (e.g., dogs bark because barking is part of what it means to be a dog).

Which properties are linked to their kinds in this principled way? A number of candidate property types have been proposed. For example, properties may be predicated generically if they fit dimensions specified by overhypotheses (e.g., each kind of animal has a characteristic diet, sound, mode of reproduction; Shipley, 1993; see Leslie, 2007, 2008). Relatedly, properties that fit with people's naïve causal theories about the relevant kinds have been shown to be accepted when predicated in generic form (Cimpian, Gelman, et al., 2010; Gelman \& Bloom, 2007). Leslie $(2007,2008)$ has proposed that properties that are especially striking or dangerous (e.g., "Sharks attack swimmers") may also be predicated generically if they are viewed as part of a kind's natural disposition (see also Cimpian, Brandone, et al., 2010). However, as Leslie (2007, 2008) points out, a property cannot be correctly predicated of a kind if there is an equally salient, positive alternative property that applies to other members of the kind. For example, "Lions are boys" may be considered false because female lions possess an equally salient alternative property (i.e., being female). In contrast, "Lions have manes" may be considered true because female lions lack this property and do not exhibit a salient competing feature. Overall, these proposals begin to address the important question regarding which sorts of properties get linked to their categories in principled ways, which get linked to their categories via prevalence alone, and why. Further work, including developmental research, on these questions is needed. In particular, research on how children learn which properties are principled versus not and on the potential role of parental input in this learning process (e.g., Gelman, Coley, Rosengren, Hartman, \& Pappas, 1998; Gelman, Taylor, \& Nguyen, 2004) may be especially informative.

To conclude, our findings demonstrate that for children, as for adults (Cimpian, Brandone, et al. 2010; Cimpian, Gelman, et al., 2010; Khemlani et al., 2007; Khemlani et al., 2009), generic meaning does not reduce to considerations of how many individuals have the relevant property. Even when children know how prevalent the relevant properties are among category members, they do not use this knowledge as the sole basis for evaluating the truth of generic statements. Rather, considerations about whether generically predicated properties are characteristic of their kind play a deciding role in children's reasoning about generic sentences. 


\section{References}

Ahn, W., Flanagan, E. H., Marsh, J. K., \& Sanislow, C. A. (2006). Beliefs about essences and the reality of mental disorders. Psychological Science, 17, 759-766.

Brandone, A. C., \& Gelman, S. A. (2009). Differences in preschoolers' and adults' use of generics about animals and artifacts: A window onto a conceptual divide. Cognition, 110, 1-22.

Carey, S. (1985). Conceptual change in childhood. Cambridge, MA: MIT Press.

Carey, S. (2009). The origin of concepts. New York: Oxford University Press.

Carlson, G. N. (1977). A unified analysis of the English bare plural. Linguistics and Philosophy, 1, 412-456.

Carlson, G. N., \& Pelletier, F. J. (Eds.). (1995). The generic book. Chicago: University of Chicago Press.

Chambers, C. G., Graham, S. A., \& Turner, J. N. (2008). When hearsay trumps evidence: How generic language guides preschoolers' inferences about unfamiliar things. Language and Cognitive Processes, 23, 749-766.

Cimpian, A., Arce, H. C., Markman, E. M., \& Dweck, C. S. (2007). Subtle linguistic cues affect children's motivation. Psychological Science, 18, 314-316.

Cimpian, A., Brandone, A. C., \& Gelman, S. A. (2010). Generic statements require little evidence for acceptance but have powerful implications. Cognitive Science, 34, 1452-1482.

Cimpian, A., Gelman, S. A., \& Brandone, A. C. (2010). Theory-based considerations influence the interpretation of generic sentences. Language and Cognitive Processes, 25, 261-276.

Cimpian, A., \& Markman, E. M. (2008). Preschool children's use of cues to generic meaning. Cognition, 107, 19-53.

Cimpian, A., Meltzer, T. J., \& Markman, E. M. (2011). Preschoolers' use of morphosyntactic cues to identify generic sentences: Indefinite singular noun phrases, tense, and aspect. Child Development, 82, 1561-1578.

Colunga, E., \& Smith, L. B. (2005). From the lexicon to expectations about kinds: A role for associative learning. Psychological Review, 112, 347-382.

Gelman, S. A. (2003). The essential child: Origins of essentialism in everyday thought. Oxford, UK: Oxford University Press.

Gelman, S. A., \& Bloom, P. (2007). Developmental changes in the understanding of generics. Cognition, 105, 166-183.

Gelman, S. A., \& Brandone, A. C. (2010). Fast-mapping placeholders: Using words to talk about kinds. Language Learning and Development, 6, 223-240.

Gelman, S. A., Coley, J. D., Rosengren, K., Hartman, E., \& Pappas, A. (1998). Beyond labeling: The role of maternal input in the acquisition of richly-structured categories. Monographs of the Society for Research in Child Development, 62(1, Serial No. 253).

Gelman, S. A., Goetz, P. J., Sarnecka, B. S., \& Flukes, J. (2008). Generic language in parent-child conversations. Language Learning and Development, 4, 1-31.
Gelman, S. A., \& Heyman, G. D. (1999). Carrot-eaters and creature-believers: The effects of lexicalization on children's inferences about social categories. Psychological Science, 10, 489-493.

Gelman, S. A., \& Raman, L. (2003). Preschool children use linguistic form class and pragmatic cues to interpret generics. Child Development, 74, 308-325.

Gelman, S. A., Taylor, M. G., \& Nguyen, S. (2004). Mother-child conversations about gender: Understanding the acquisition of essentialist beliefs. Monographs of the Society for Research in Child Development, 69(1, Serial No. 275).

Gelman, S. A., Waxman, S., \& Kleinberg, F. (2008). The role of representational status and item complexity in parent-child conversations about pictures and objects. Cognitive Development, 23, 313-323.

Gilson, C., \& Abelson, R. P. (1965). The subjective use of inductive evidence. Journal of Personality and Social Psychology, 2, 301-310.

Gopnik, A., \& Meltzoff, A. N. (1997). Words, thoughts, and theories. Cambridge, MA: Bradford Books/MIT Press.

Gopnik, A., \& Schulz, L. (2004). Mechanisms of theory formation in young children. Trends in Cognitive Sciences, 8, 371-377.

Gopnik, A., \& Schulz, L. (Eds.). (2007). Causal learning: Psychology, philosophy, and computation. New York: Oxford University Press.

Hollander, M. A., Gelman, S. A., \& Star, J. (2002). Children's interpretations of generic noun phrases. Developmental Psychology, 38, 883-894.

Keil, F. (1989). Concepts, kinds, and cognitive development. Cambridge, MA: Bradford Book/MIT Press.

Keil, F. C. (2006). Explanation and understanding. Annual Review of Psychology, 57, 227-254.

Khemlani, S., Leslie, S. J., \& Glucksberg, S. (2009). Generics, prevalence, and default inferences. Proceedings of the 31st Annual Conference of the Cognitive Science Society. Austin, TX: Cognitive Science Society.

Khemlani, S., Leslie, S. J., Glucksberg, S., \& Rubio-Fernandez, P. (2007). Do ducks lay eggs? How humans interpret generic assertions. Proceedings of the 29th Annual Conference of the Cognitive Science Society. Nashville, TN: Cognitive Science Society.

Leslie, S. J. (2007). Generics and the structure of the mind. Philosophical Perspectives, 21, 375-405.

Leslie, S. J. (2008). Generics: Cognition and acquisition. Philosophical Review, 117, 1-49.

Markman, E. M. (1989). Categorization and naming in children: Problems in induction. Cambridge: Bradford Book/ MIT Press.

Murphy, G. L. (2002). The big book of concepts. Cambridge, MA: MIT Press.

Piaget, J. (1952). The origins of intelligence in children. New York: International University Press.

Prasada, S. (2000). Acquiring generic knowledge. Trends in Cognitive Sciences, 4, 66-72. 
Prasada, S., \& Dillingham, E. M. (2006). Principled and statistical connections in common sense conception. Cognition, 99, 73-112.

Prasada, S., \& Dillingham, E. M. (2009). Representation of principled connections: A window onto the formal aspect of common sense conception. Cognitive Science, 33, 401-448.

Reynaert, C. C., \& Gelman, S. A. (2007). The influence of language form and conventional wording on judgments of illness. Journal of Psycholinguistic Research, 36, 272-295.

Rips, L. J., \& Collins, A. (1993). Categories and resemblance. Journal of Experimental Psychology: General, 122, 468-486.

Saffran, J. R., Aslin, R. N., \& Newport, E. L. (1996). Statistical learning by 8-month-olds infants. Science, 274, 1926-1928.

Shipley, E. F. (1993). Categories, hierarchies, and induction. Psychology, Learning, and Motivation, 30, 265-301.

Sloutsky, V. M., \& Fisher, A. V. (2004). Induction and categorization in young children: A similarity-based model. Journal of Experimental Psychology: General, 133, 166-188.

Sloutsky, V. M., Kloos, H., \& Fisher, A. V. (2007). When looks are everything: Appearance similarity versus kind information in early induction. Psychological Science, 18, 179-185.

Smith, L. B., Jones, S. S., \& Landau, B. (1996). Naming in young children: A dumb attentional mechanism? Cognition, 60, 143-171.

Sobel, D. M., \& Kirkham, N. Z. (2007). Interactions between causal and statistical learning. In A. Gopnik \& L. E. Schulz (Eds.), Causal learning: Psychology, philosophy, and computation (pp. 139-153). New York: Oxford University Press.

Tenenbaum, J. B., Griffiths, T. L., \& Kemp, C. (2006). Theory-based Bayesian models of inductive learning and reasoning. Trends in Cognitive Sciences, 10, 311-318.

Wellman, H. M., \& Gelman, S. A. (1998). Knowledge acquisition. In D. Kuhn \& R. Siegler (Eds.), Handbook of child psychology: Vol. 2. Cognition, perception, and language (4th ed., pp. 523-573). Hoboken, NJ: Wiley. 